\title{
Flow Analysis and Modeling of Magneto-Rheological Fluids
}

\author{
H.K.Gao \\ Research Centre for Intelligent Materials and Structures \\ (CIMS) \\ Huaqiao University
}

\author{
D.S. Qu \\ School of Automation Science \& Engineering \\ South China University of Technology
}

\author{
F.Yang \\ Research Centre for Intelligent Materials and Structures (CIMS) \\ Huaqiao University
}

\begin{abstract}
The Bingham-plasticmodel has been widely used to predict the post-yield behavior of Electro-Rheological(ER) and Magneto-Rheological(MR) fluids. However, as the plastic viscosity is not constant under conditions of different flow velocity and applied field (Electric/Magnitude), the phenomenon of shear thinning or shear thickening exists in the flow of ER/MR fluids. At this condition, the Bingham-plastic may not be an accurate model to predict the behavior of ER/MR fluids.In this study, the equations of motion of the MR fluids based on parallel plates and annulus duct models has been established, and the general solutions has been obtained based on the Herschel-Buckley model. The analysis of the impacts of the external magnetic field strength applying on the MR fluid has also been conducted. Therelated velocity profile, flow rate curves have been presented in the numerical example to verify the validity of the proposed methodologies.
\end{abstract}

Keywords-electro-rheological (ER) fluid;magneto-rheological (MR) fluid;parallel plates; annulus duct;bingham-plastic model; herschel-bulkley model

\section{INTRODUCTION}

Since the 1990s, the investigation of the application of MR fluids, proposed by Rabinow[1] in the 1940s, has become a research interest.Among all of the research regarding the application of MR fluids, the MR damper may be the most popular research topic, as it can provide controllable damping within related small dimension and larger working temperature ranger with fail-safety properties[2]. However, most of research papers regarding the MR damper are focused on the modelling and control methodologies of the MR damper[3].

The Newtonian and Bingham plastic models have been widely utilized to describe the flow behavior characteristics of ER/MR fluids[4], and was applied to the design of ER/MR dampers. However, as the phenomenon of shear thinning or shear thickening exists in the flow of ER/MR fluids, the Bingham-plastic may not be an accurate model to predict the behavior of ER/MR fluids. Therefore, Herschel-Bulkley model has also been utilized to describe the flow properties of ER/MR fluids by some researchers[5].
This study presents the general solution of the flow model of MR fluids through the parallel plates and annulus duct based on the Herschel-Bulkley model. And then, the influence of the applied magnitude field to the flow has also been conducted. Numerical example has been provided to verify the validity of the established methodologies, and the research presented in this paper is the fundamental for design, modelling and testing of MR dampers.

\section{MOdELliNG OF FLOW MODEL USING HERSCHEL- BULKLEY MODEL}

The Herschel-Bulkley constitutive equation, presented in eqn. (1),indicates the relationship between the shear stress and shear strain of Non-Newtonian fluid [6].

$$
\begin{cases}\tau=\tau_{y}+|\eta \dot{\gamma}|^{1 / n} & |\tau| \geq \tau_{\mathrm{y}} \\ \dot{\gamma}=0 & |\tau| \leq \tau_{\mathrm{y}}\end{cases}
$$

Where $\tau$ and $\tau_{\mathrm{y}}$ are shear and yield stress perpendicular to the flow direction; $\dot{\gamma}$ is shear strain rate;nand $\eta$ isthe flow behavior and consistency index,respectively.It should be noted that comparing to the Bingham model, the HerschelBulkley model accounts forthe post-yield shear thinning or thickening behavior ofMR fluids, and can describe the pseudo-plastic or shear thinning behavior $(n>1)$, the Bingham model $(\mathrm{n}=1)$, and the shear thickening behavior $(\mathrm{n}<1)$.

\section{A. Flow between Flat Parallel Plates}

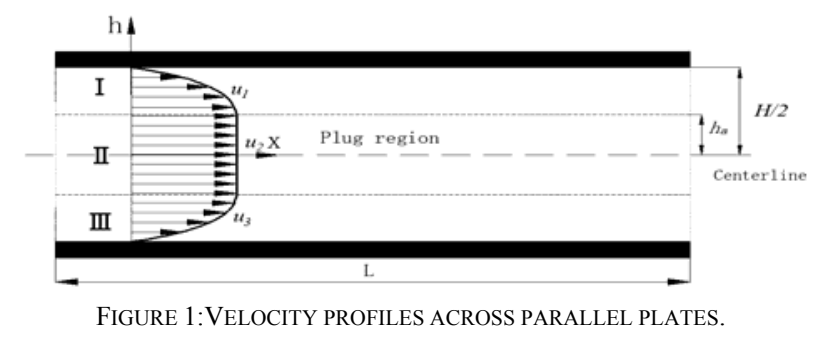

Fig.1illustrates the MR fluids flow through parallel plates along the direction of x.L and Hrepresent the length of the plate and the distance between two parallel plates, respectively.The velocity profile can be divided three 
sections named region I, II, III, and the corresponding velocities are u1, u2, u3, respectively. Here, it should be noted that the region II is plug region in which the shear stress is equal to zero at $\mathrm{h}=\mathrm{ha}$.

Between centerline and the parallel plate surface fluids flow through the gap have a value of linear velocity $u$, and consider stress distribution from zero at the centerline to $\tau_{\mathrm{w}}$ at the plates surface, hence [6]:

$$
\tau=\frac{\Delta P h}{L} \text { for } 0 \leq h \leq H / 2
$$

and the value of velocity gives:

$$
\begin{gathered}
u_{1}=\int_{0}^{u} d u=\int_{H / 2}^{h}\left(\frac{d u}{d h}\right) d h=\int_{h}^{H / 2}\left(-\frac{d u}{d h}\right) d h \text { for } \\
h_{a} \leq h \leq H / 2
\end{gathered}
$$

Considering the boundary conditions for region I,IIandIII, the velocity profile can be expressed as:

$$
\begin{gathered}
u_{1}=\frac{L}{\eta(n+1) \Delta P}\left[\left(\frac{\Delta P H}{2 L}-\tau_{y}\right)^{n+1}-\left(\frac{\Delta P h}{L}-\tau_{y}\right)^{n+1}\right] \\
u_{2}=\frac{L}{\eta(n+1) \Delta P}\left[\left(\frac{\Delta P H}{2 L}-\tau_{y}\right)^{n+1}-\left(\frac{\Delta P \cdot h_{a}}{L}-\tau_{y}\right)^{n+1}\right]
\end{gathered}
$$

The total volumetric flow rate $\mathrm{Q}$ at the parallel gap can be expressed as:

$$
Q=Q_{1}+Q_{2}+Q_{3}=\int_{h_{a}}^{H / 2} u_{1} \cdot d h+\int_{h_{a}}^{H / 2} u_{2} \cdot d h+\int_{0}^{h_{a}} u_{3} \cdot d h
$$

Substituting eqns.(4) and (5) into eqn. (6), one can obtain:

$$
Q=\frac{W H^{2}\left(\frac{\Delta P H}{2 L}-\tau_{y}\right)^{n+1}}{2(n+1) \eta \tau_{w}}\left[1-\frac{\left(\frac{\Delta P H}{2 L}-\tau_{y}\right)}{(n+2) \tau_{w}}\right]
$$

\section{B. Flow through a Concentric Annulus Duct}

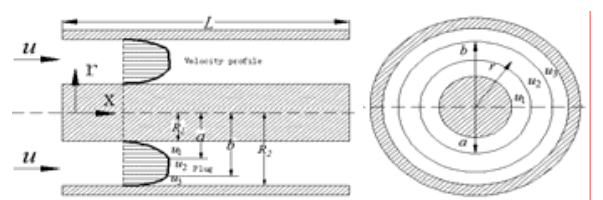

FIGURE 2:VELOCITY PROFILE FLOW IN AN ANNULUS DUCT.

Fig. 2 illustrates the velocity profile of MR fluids flow through an annulus duct along the direction $\mathrm{x}$. R1and R2 represent the inner and outer radius, respectively.aandbrepresent the plug boundaries, and the corresponding velocities are illustrated asu1, u2, u3 .

Because of the axis in the center of the circular tube, so the shear stress takes minus sign when $\mathrm{R} 1<\mathrm{r}<\mathrm{a}$, similarly it takes plus sign when $\mathrm{b}<\mathrm{r}<\mathrm{R} 2$. The shear stress on this surface is [6]:

$$
\begin{gathered}
\tau=\tau_{y}+(\eta \dot{\gamma})^{1 / n} \text { for } R_{1}<r<a \\
\tau=\tau_{y}+(\eta(-\dot{\gamma}))^{1 / n} \text { for } b<r<R_{2}
\end{gathered}
$$

The velocity profile of the flow can be derived and expressed as:

$$
\left\{\begin{array}{l}
u_{1}=\int_{R_{1}}^{r} \frac{1}{\eta}\left(-\frac{\Delta P r}{2 L}-\frac{A}{r}-\tau_{y}\right)^{n} d r \\
u_{2}=\int_{R_{2}}^{r} \frac{-1}{\eta}\left(\frac{\Delta P r}{2 L}+\frac{A}{r}-\tau_{y}\right)^{n} d r \\
u_{3}=\int_{R_{1}}^{a} \frac{1}{\eta}\left(-\frac{\Delta P r}{2 L}-\frac{A}{r}-\tau_{y}\right)^{n} d r
\end{array}\right.
$$

With the boundary condition of velocity profile as:

$$
u_{3}=\left.u_{1}\right|_{r=a}=\left.u_{2}\right|_{r=b}
$$

The total volumetric flow rateQ=Q1+Q2+Q3, where,

$$
\begin{gathered}
Q_{1}=\pi\left(a^{2} \int_{R_{1}}^{a} \dot{\gamma}_{1} d r-\int_{R_{1}}^{a} \dot{\gamma}_{1} r^{2} d r\right) \\
Q_{2}=\pi\left(\int_{R_{2}}^{b} \dot{\gamma}_{2} r^{2} d r-b^{2} \int_{R_{2}}^{b} \dot{\gamma}_{2} d r\right) \\
Q_{3}=\pi\left(b^{2}-a^{2}\right) \cdot u_{3}=\pi\left(b^{2}-a^{2}\right) \cdot \int_{R_{1}}^{a} \dot{\gamma}_{1} d r
\end{gathered}
$$

\section{NUMERICAL RESULTS}

In this section, the investigation of the flow properties for parallel plates and annulus ductsbased on the HerschelBulkley model will be presented. And then, the studies will be extended to the effectiveness of the applied magnitude field to the flow properties of a typical type of MR fluid. The geometrical parameters of the parallel plate and annulus duct have been illustrated in Table 1 .

TABLE 1: GeOMETRIC SPECIFICATIONS OF COMPUTER SimUlation.

\begin{tabular}{cccc}
\hline Parallel plates & Value(m) & $\begin{array}{l}\text { Annulus } \\
\text { duct }\end{array}$ & Value(m) \\
\hline Length $\boldsymbol{L}$ & 0.1 & $\begin{array}{c}\text { Inner } \\
\text { radius, } R_{1} \\
\text { Outer }\end{array}$ & 0.03 \\
Wide, $\boldsymbol{W}$ & 0.25 & $\begin{array}{c}\text { radius, } R_{2} \\
\text { Length, } L\end{array}$ & 0.05 \\
Distance, $\boldsymbol{H}$ & 0.02 & 0.1 \\
\hline
\end{tabular}

\section{A. Flow Properties Analysis}

To verify the validity of the established modelling procedure, as shown in Section 2, the plug boundaries, velocity profile and flowrateproperties has been obtained and illustrated in figs. 3,4,and 5, respectively. Here, it should be noted that in figs. 3,4 and 5 the value of $\tau_{y}$, and index nand $\eta$ have been selected as $2.865 \mathrm{kPa}, 1.26$ and 3.89 , respectively, as those provided by Choi et al. [5]. 


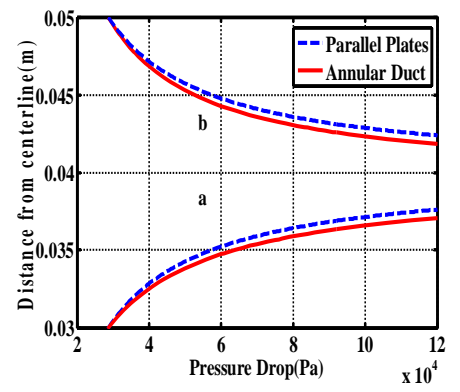

FIGURE 3: PLUG BOUNDARIES A ANDB.

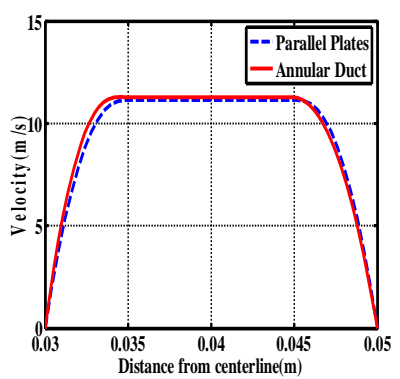

FigURE 4:VELOCITY PROFILE $(\Delta \mathrm{P}=5.5 \times 104 \mathrm{PA})$.

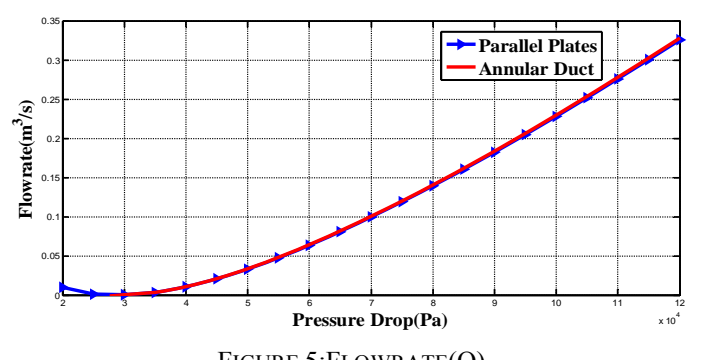

FIGURE 5:FLOWRATE(Q).

From figs. 3-5,it can be found that: (1) with the increase of pressure drop $(\Delta \mathrm{P})$, the plug area for both parallel plates and annulus duct models are become narrow, and the velocity (flow rate) become large; (2) There are no significant difference between parallel plates and annulus duct models for the flow rate, especially for the pressure drop bigger than $3 \times 104$. The results presented in this Section have been compared with those shown in available literatures, such as that presented by Choi et al. [5], and perfect match can be found. Following, the annulus duct model will be utilized to investigate the influence to the fluid behavior index and applied magnitude field to the flow behavior.

\section{B. The Influence of the Fluid BehaviorIndex (N)}

As mentioned before, the Herschel-Bulkley model can describe the pseudo-plastic or shear thinning behavior $(n>1)$, the Bingham model $(n=1)$, and the shear thickening behavior $(\mathrm{n}<1)$. In this section, the influence of the fluid behavior index (n) to the shear stress $\tau$, which can directly affect of the flow properties. Fig. 6 illustrates the relationship between shear stress $(\tau)$ with shear rate $(\dot{\gamma})$ with different fluid behavior index (n) under the yield stress $\tau_{\mathrm{y}}$ equal to $2415 \mathrm{~Pa}$. fig. 7 shows the relationship of the shear stress $(\tau)$ with fluid behavior index (n) under the yield stress $\tau_{\mathrm{y}}$ equal to $2415 \mathrm{~Pa}$ and shear rate $\dot{\gamma}$ to $10 \mathrm{~K} 1 / \mathrm{sec}$.

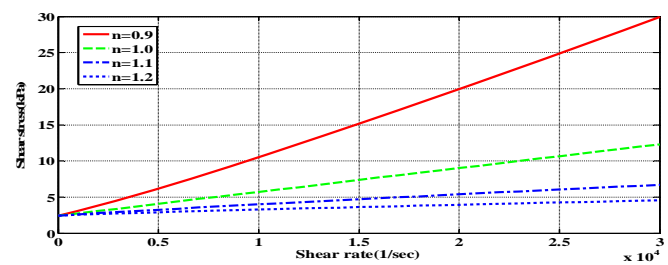

FIGURE 6:THE RELATIONSHIP BETWEEN SHEAR STRESS WITH SHEAR RATE UNDER DIFFERENT FLUID BEHAVIOR INDEX (N).

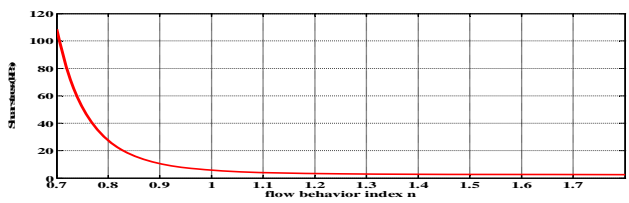

FIGURE 7:THE RELATIONSHIP OF THE SHEAR STRESS (T) WITH FLUID BEHAVIOR INDEX $(\mathrm{N})$.

From figs. 6 and 7, it can be found that shear stress change significantly when the fluid behavior index (n) smaller than 1, and almost stable when the $n$ bigger than 1.1. Therefore, one can easily make conclusion that the HerschelBulkley model with $n>1$ can provide more accurate result to estimate the flow behaviour of MR fluid, which has typical thickening properties, than the Bingham model $(n=1)$. Typically, the fluid behaviorindex(n) for MR fluid is selected higher than 1.1, as it can provide stable result, as shown in fig. 7

C. The Influence of the Applied Magnetic Field Tomr Fluids

In this section, the flow properties of MR fluids will be investigated based on the methodologies, established in Section 2, and the study will also be extended to the influence of the applied magnetic field to the flow properties of the MR fluid, which is the fundamental of the optimal design of MR damper or any application related to the flow working model of MR fluids.

Here, the 132LD type MR fluids from LORD Company has been selected, and Therelationship between the applied current (directly affecting the applied magnetic field) with the yield $\operatorname{stress} \tau_{\mathrm{y}}$ can be expressed as [5]:

$$
\tau_{y}=2.7 \times 10^{4} I^{1.5}
$$

Following the same procedure, presented in Section 2, one can get the plug boundaries, velocity profile and flowrateproperties under different input current (magnetic field), as those illustrated in figs 8-10. Here, it should be noted that theconsistencyindexpis selected as 0.33 Pa.sec, and $n$ equal to 1.1 . 


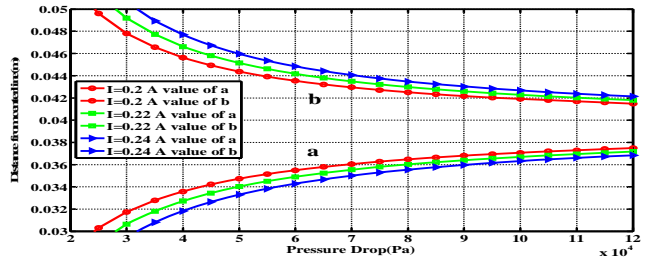

FIGURE 8:THEPLUG BOUNDARIES A ANDBUNDER DIFFERENT INPUT CURRENTI.

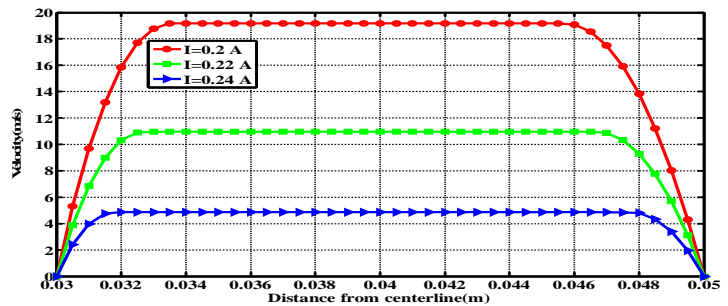

FIGURE 9:VELOCITY PROFILE UNDER DIFFERENT INPUT CURRENT I WITH $\triangle \mathrm{P}=4 \times 104 \mathrm{PA}$.

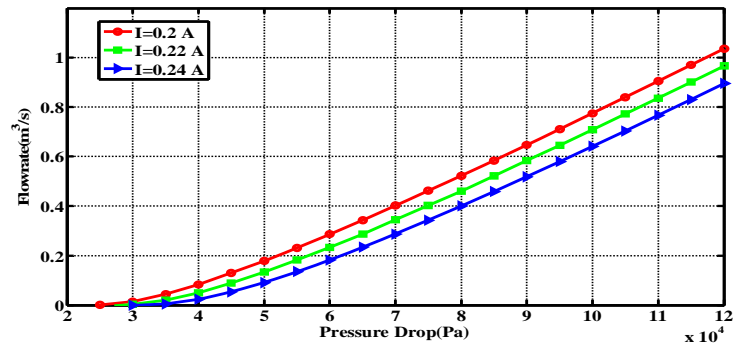

FIGURE 10:FLOWRATE (Q) UNDER DIFFERENT INPUTCURRENTI.

It can be found from Figs 8-10 that with the increasing of the input current (magnitude field) (1) the plug boundaries became large, and the maximum velocity dropped significantly (the velocity at $0.2 \mathrm{~A}$ input current is around 1.7 times that of $0.22 \mathrm{~A}$, and about 3.8 times that of $0.24 \mathrm{~A}$ ); (2) the flow rate also dropped, but not so significantly change as the increasing of the plug boundaries; (3) the flowratedecreases (the flowrate for the current $0.2 \mathrm{~A}$ is nearly 1.7 times that at the current $0.22 \mathrm{~A}$, and 3.6 times that at the current $0.24 \mathrm{~A}$, under $\Delta \mathrm{P}=4 \times 104 \mathrm{~Pa}$; the flowrateof the current $0.2 \mathrm{~A}$ is nearly 1.7 times that at the current $0.22 \mathrm{~A}$, and 3.6 times that at the current $0.24 \mathrm{~A}$ under $\Delta \mathrm{P}=1.1 \times 105 \mathrm{~Pa}$ ).

\section{CONCLUSION}

This study presents a general analytical procedure to investigate the flow behavior of the MR fluids under both parallel plates and annulus duct models using the HerschelBulkleyfluidsmodel. The results show that: (1) There are no significant difference between parallel plates and annulus duct models for the flow rate, especially for high pressure drop; (2) the Herschel-Bulkley model with $n>1$ can provide more accurate result to estimate the flow behaviour than the Bingham model (Herschel-Bulkley model with $\mathrm{n}=1$ ); (3) with the increase of the applied input current (applied magnitude field), the flow rate drops a lot, and then the Herschel-Bulkley model with $n>1$ can provide superior result to estimate the flow behaviour of MR fluid, which has typical thickening properties.

\section{ACKNOWLEDGEMENTS}

Support from the National Science Foundation of China (NSFC): 61374144 are gratefully acknowledged.

\section{REFERENCES}

[1] Rabinow, J., The Magnetic Fluid Clutch.AIEE Trans., 67 1308$1315,1948$.

[2] Yang. F, Sedaghati R and Esmailizedah E. Development of LuGre friction model for large-scale Magneto-Rheological fluid dampers, Journal of Intelligent Material Systems and Structures, 20(8): 923937, 2009.

[3] Yang. F, Sedaghati R and Esmailizedah E. Optimal Vibration Suppression of Structures UnderRandom Base Excitation Using SemiActive Mass Damper, ASME Journal of Vibration and Acoustics, Article Number: 041002, 2010 .

[4] Yang G, Spencer Jr.BF, Carlson JD, Sain MK. Large-scale MR fluid Dampers: modeling, and dynamic performance considerations. Engineering Structures, 24(3): 309-23, 2002.

[5] Lee D.Y., Choi Y.T. and Wereley, N.M, Performance analysis of ER/MR impact damper systems using HerschelBulkleymodel.Journal of Intelligent Material Systems and Structures, 13, 525-31,2002.

[6] Skelland AHP, Non-Newtonian flow and heat transfer. United States of America: John Wiley and Sons, 196. 